\title{
Improving the affinity of fibroblasts for bacterial cellulose using carbohydrate-binding modules fused to RGD
}

\author{
F. K. Andrade, S. M G. Moreira, L. Domingues, F. M. P. Gama \\ IBB-Institute for Biotechnology and Bioengineering, Centre of Biological Engineering, Universidade do Minho, \\ Campus de Gualtar, 4710-057 Braga, Portugal
}

Received 11 December 2007; revised 16 April 2008; accepted 3 June 2008

Published online 22 January 2009 in Wiley InterScience (www.interscience.wiley.com). DOI: 10.1002/jbm.a.32284

\begin{abstract}
The attachment of cells to biomedical materials can be improved by using adhesion sequences, such as Arg-Gly-Asp (RGD), found in several extracellular matrix proteins. In this work, bifunctional recombinant proteins, with a Cellulose-Binding Module (CBM), from the cellulosome of Clostridium thermocellum and cell binding sequences-RGD, GRGDY-were cloned and expressed in E.coli. These RGD-containing cellulose binding proteins were purified and used to coat bacterial cellulose fibres. Its effect on the cell adhesion/biocompatibility properties was tested using a mouse embryo fibroblasts culture. Bacterial cellulose (BC) secreted by Gluconacetobacter xylinus (=Acetobacter xylinum) is a material with unique properties and promis-
\end{abstract}

ing biomedical applications. CBMs adsorbs specifically and tightly on cellulose. Thus, they are a useful tool to address the fused RGD sequence (or other bioactive peptides) to the cellulose surface, in a specific and simple way. Indeed, fibroblasts exhibit improved ability to interact with bacterial cellulose sheets coated with RGD-CBM proteins, as compared with cellulose treated with the CBM, that is, without the adhesion peptide. The effect of the several fusion proteins produced was analyzed. (C) 2009 Wiley Periodicals, Inc. J Biomed Mater Res 92A: 9-17, 2010

Key words: bacterial cellulose; CBM, fibroblasts; RGD; cell adhesion

\section{INTRODUCTION}

The fundamental premise of tissue engineering is to develop tissue substitutes to restore or improve the function of diseased or damaged human tissues. Many biomaterials have been studied as scaffolds, in which the cells and/or growth factors can be seeded, cultured, and then, implanted to induce and direct the growth of new, healthy tissue.

The primary function of a scaffold is tissue conduction and, therefore, it must allow cell attachment, migration onto or within the scaffold, cell proliferation and cell differentiation. It must also provide an environment where the cells conserve their phenotype. ${ }^{1}$ The successful development of tissue engineering scaffolds requires proper substrates for cell survival and differentiation. The attachment of cells to the biomedical materials can be improved by using adhesion molecules. These molecules, present in the extracellular matrix proteins, regulate the adhesion,

Correspondence to: F. M. P. Gama; e-mail: fmgama@deb. uminho.pt

Contract grant sponsor: FUNCARB POCTI; contract grant number: $\mathrm{BIO} / 45356 / 2002$

(C) 2009 Wiley Periodicals, Inc. migration and growth of cells, by binding to integrin receptors located on the outer cellular membranes. ${ }^{2,3}$ The tripeptide motif RGD was identified 23 years ago by Pierschbacher and Rouslahti as the minimal essential cell adhesion peptide sequence in fibronectin. ${ }^{4}$ Since then, cell adhesive RGD sites were identified in many other extracellular matrix (ECM) proteins, including vitronectin, fibrinogen, von Willebrand factor, collagen, laminin, osteopontin, tenascin, and bone sialoprotein as well as in membrane proteins, in viral and bacterial proteins, and in snake venoms (neurotoxins and disintegrins). ${ }^{5}$ The RGD sequence is by far the most effective and most often employed peptide sequence used to stimulate cell adhesion on synthetic surfaces, due to its widespread distribution and use throughout the organism, its ability to address more than one cell adhesion receptor, and its biological impact on cell anchoring, behavior, and survival. ${ }^{6}$ Incorporation of biomimetic adhesion sites can be used to promote cell adhesion and migration on or within bioactive materials. The selection of which type of cells adheres to a material and their spatial distribution can also be controlled through the selection of the adhesion sites that are incorporated into the bioactive material. ${ }^{7}$

In the present study, we analyzed the adhesion of mouse embryo fibroblasts on bacterial cellulose (BC). 


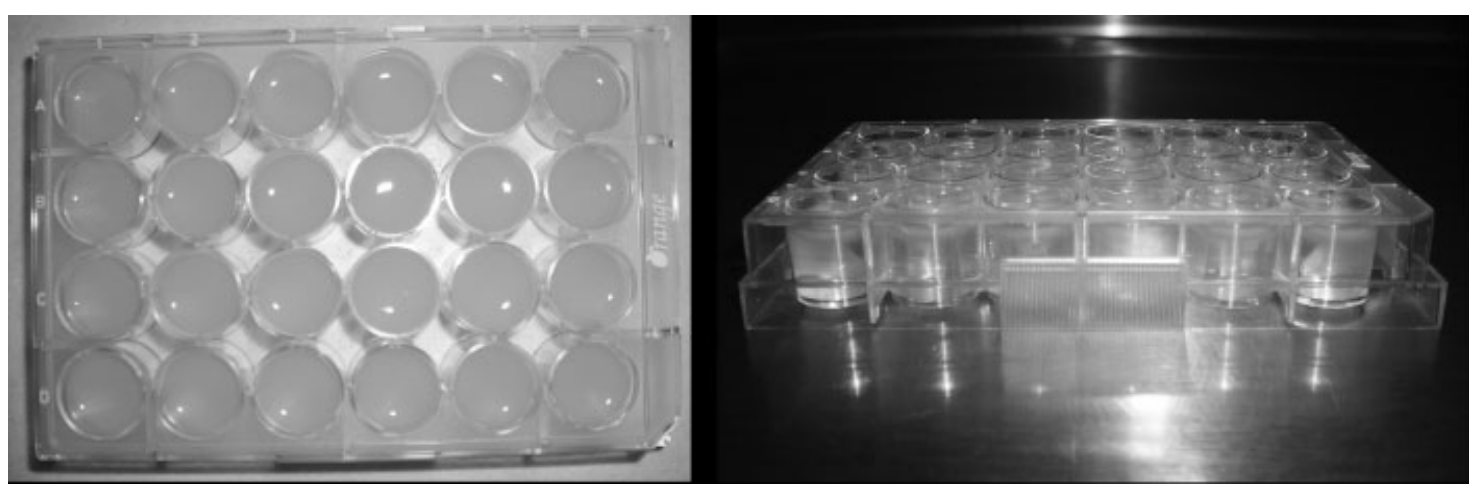

Figure 1. Gluconacetobacter xylinus (ATCC 53582) cultured on liquid Hestrin-Schramm medium after 7 days. The medium was inoculated with the culture and added to the 24 -well polystyrene plate $(1 \mathrm{~mL} /$ per well $)$ and incubated statically at $30^{\circ} \mathrm{C}$.

$\mathrm{BC}$ is secreted by Gluconacetobacter xylinus (=Acetobacter xylinum). It is a material with unique properties, including high water holding capacity, high crystallinity, ultrafine fiber network, and high tensile strength. ${ }^{8}$ Although chemically identical to plant cellulose, it has a unique fibrillar nanostructure, which determines its extraordinary physical and mechanical properties, characteristics which are quite promising for modern medicine and biomedical research. ${ }^{9}$ For example, membranes of bacterial cellulose were used as an artificial skin for temporary covering of wounds and bacterial cellulose tubes were produced as substitution material for blood vessels. BC was also successfully used in periodontal treatments and applied as replacement for dura mater (a membrane that surrounds brain tissue). ${ }^{8,9}$

A method for producing chimeric proteins, RGDCBM containing a cellulose-binding module (CBM), was developed. CBMs are discrete protein modules found in a large number of carbohydrolases and a few nonhydrolytic proteins. ${ }^{10}$ A cellulose-binding domain family III from the cellulosomal-scaffolding protein A of the bacteria Clostridium thermocellum, was used in the present work. ${ }^{11}$ The main goal was the functionalization of bacterial cellulose aiming its utilization as scaffold in tissue engineering. There are several methodologies to bind adhesion molecules to the biomaterials. However, the use of a $\mathrm{CBM}$, as proposed in this work, is a simple way to direct bioactive peptides to the cellulose surface, avoiding the use of complex peptide chemical grafting. Furthermore, the adsorption of this CBM to BC is specific and very stable. Indeed, the removal of CBM from cellulose can be achieved only using denaturant agents. ${ }^{10}$ Thus, the coating of BC with recombinant proteins is stable enough for its practical application in the designed of biomaterials.

The genes encoding these CBM-containing chimeric proteins were cloned, and the proteins expressed and purified. Polystyrene surfaces and bacterial cellulose sheets where "coated" with these
RGD-containing proteins, and then used in adhesion/biocompatibility tests, using a mouse embryo fibroblasts culture. Fibroblasts were selected as model animal cells to test our strategy of bacterial cellulose modification, while the polystyrene plate was used as a model surface to test the recognition of the RGDs sequences by the integrins on cells membrane.

\section{MATERIALS AND METHODS}

\section{Production of bacterial cellulose}

Gluconacetobacter xylinus (ATCC 53582) purchased from the American Type Culture Collection was grown in Hestrin-Schramm medium, $\mathrm{pH}$ 5.0. The medium was inoculated with the culture, added to the 24-well polystyrene plate $\left(1 \mathrm{~mL} /\right.$ per well) and incubated statically at $30^{\circ} \mathrm{C}$, for 7 days. BC pellicles were purified by $4 \%$ SDS treatment at $70^{\circ} \mathrm{C}$, for $12 \mathrm{~h}$ followed by $4 \% \mathrm{NaOH}$ at $70^{\circ} \mathrm{C}$, for $90 \mathrm{~min}$. Samples were autoclaved and stored in PBS pH 7.4, at $4^{\circ} \mathrm{C}$, prior to use (Fig. 1).

\section{Construction of a gene fusion encoding the adhesion peptide and a CBM}

The pET21a vector (Novagen) with the CBM from C. thermocellum and a N-terminal linker cloned in the NheI and XhoI restriction sites was used as template for amplification of the fused genes. The nucleotide sequence of each peptide was determined and the sequences optimized for E. coli expression. Peptides were then amplified by PCR with the enzyme Vent DNA polymerase (New England Biolabs) using primers presented in Table I. Primers included NheI and XhoI restriction sites, which are shown in bold. PCRs were performed as follows: preheating at $95^{\circ} \mathrm{C}$ for $2 \mathrm{~min}, 40$ cycles at $95^{\circ} \mathrm{C}$ for $45 \mathrm{~s}, 53^{\circ} \mathrm{C}$ for $45 \mathrm{~s}$, and $72^{\circ} \mathrm{C}$ for $45 \mathrm{~s}$, followed by an elongation at $72^{\circ} \mathrm{C}$ for 10 $\mathrm{min}$. The DNA recombinant coding sequences were cloned in pET21a using E. coli XL1 Blue (Strategene) as cloning strain. Clones containing the recombinant genes were identified by restriction enzyme analysis. Amplified fragments 
TABLE I

PCR Primers with the Restriction Sites NheI (GCTAGC) and XhoI (CTCGAG) Used for Cloning the Gene Fusions Encoding the Adhesion Peptide with CBM

\begin{tabular}{ll}
\hline Recombinant Protein Name & \multicolumn{1}{c}{ Primers } \\
\hline RGD/CBM & Fwd: 5'-CTA GCT AGC AGA GGT GAT ACA CCG ACC AAG GGA G-3' \\
& Rev: 5'-CAC CTC GAG TTC TTT ACC CCA TAC AAG AAC-3' \\
GRGDY/CBM & Fwd: 5'-CTA GCT AGC GGT AGA GGT GAT TAT ACA CCG ACC AAG GGA G-3' \\
& Rev: 5'-CAC CTC GAG TTC TTT ACC CCA TAC AAG AAC-3' \\
RGD/CBM/RGD & Fwd: 5'-CTA GCT AGC AGA GGT GAT ACA CCG ACC AAG GGA G-3' \\
& Rev: 5'-CAC CTC GAG ATC ACC TCT CGG TTC TTC AGG TTC TGT ACC GCC CGG CGG \\
GRGDY/CBM/GRGDY & CGT TCC TTC TTT ACC CCA TAC AAG AAC-3' \\
& Fwd: 5'-CTA GCT AGC GGT AGA GGT GAT TAT ACA CCG ACC AAG GGA G-3' \\
& Rev: 5'-CAC CTC GAG ATA ATC ACC TCT ACC CGG TTC TTC AGG TTC TGT ACC GCC CGG \\
& CGG CGT TCC TTC TTT ACC CCA TAC AAG AAC-3' \\
\hline
\end{tabular}

were sequenced to ensure that no mutations had occurred in the PCRs. The recombinant derivates were then digested with NheI and XhoI and the excised products were cloned into the expression vector pET21a (Novagen), previously digested with the same restriction enzymes. This vector carries a T7lac promoter and includes a C-terminal His6tag in the recombinant proteins to allow the purification. The sequence encoding the adhesion peptide (RGD or GRGDY) was annealed with the CBM at the N-terminal, through the N-terminal linker from the CBM containing 40 amino acids. To introduce another adhesion sequence at the C-terminal of the CBM, a linker containing 12 amino acids was inserted between the C-terminal of the CBM and the new adhesion sequence (Table I, Fig. 2).

\section{Production and purification of recombinant proteins}

High-level expression studies and protein production were carried out in E. coli BL21 (DE3) cells grown at $37^{\circ} \mathrm{C}$ in LB medium supplemented with ampicillin at $100 \mu \mathrm{g} /$ $\mathrm{mL}$. Cultures were induced at $\mathrm{OD}_{595}=0.5$ with IPTG $(1$ $\mathrm{mM})$. Five hours after induction the cells were separated from the culture medium by centrifugation at $13,000 \mathrm{~g}$. Then, cell pellet was ressuspended with buffer A $(20 \mathrm{mM}$ Tris, $20 \mathrm{mM} \mathrm{NaCl}, 5 \mathrm{mM} \mathrm{CaCl} 2 \mathrm{pH} 7.4$ and phenylmethylsulfonylfluoride (PMSF) $0.1 \mathrm{mM}$ ) and lysed by sonication. Imidazole was added to a final concentration of $40 \mathrm{mM}$ and the $\mathrm{pH}$ was adjusted to 7.4. The cell-free extract (soluble fraction) was then, collected by centrifugation at $15,000 \mathrm{~g}$ for $30 \mathrm{~min}$, at $4^{\circ} \mathrm{C}$ and the His6-tagged recombi- nant proteins purified by immobilized metal ion affinity chromatography, using a $5 \mathrm{~mL}$ nickel His-Trap column (GE Healthcare). Following purification, the proteins were dialyzed against buffer $\mathrm{A}$ and stored at $-20^{\circ} \mathrm{C}$, prior to use. Samples from purification's different steps and pure protein were analyzed by SDS-PAGE using a $12 \%(\mathrm{~W} / \mathrm{V})$ acrylamide gel, Coomassie stained.

\section{Interaction of recombinant proteins with the cells}

To demonstrate the interaction of the recombinant RGD with cell membrane, the recombinant proteins were chemically conjugated with isothiocyanate (FITC). The fluorescent proteins were added to the wells of 96-well polystyrene plates and the fibroblasts were added in $200 \mu \mathrm{L}$ of DMEM medium with/without serum. The plates were incubated for $1 \mathrm{~h}$ at $37^{\circ} \mathrm{C}$, in atmosphere of $5 \% \mathrm{CO}_{2}$ and 95\% humidified air. The cells were observed through fluorescence microscopy.

Effect of the recombinant proteins on the adhesion and spreading of fibroblasts

The mitochondrial activity of the cultured cells was determined using a colorimetric assay, which is related to cell viability. The MTS [3-(4,5-dimethylthiazol-2-yl)-5-(3carboxymethoxyphenyl)-2-(4-sulfophenyl)-2H-tetrazolium] assay was performed as follows: the purified recombinant proteins were added to the wells of the 96-well polystyrene plates $(0.05 \mathrm{mg}$ of protein/per well), and left adsorb-

\begin{tabular}{|l|l|l|l|l|}
\hline RGD/ GRGDY & Limkerl (40 an) & CBM & His-Tag & Step \\
\hline
\end{tabular}

(A)

\begin{tabular}{|l|l|l|l|l|l|l|}
\hline RGD/ GRGDY & Limkerl (40 aa) & CBM & Limker (12 aa) & RGD / GRGDY & His-Tag & Stop \\
\hline
\end{tabular}

(B)

Figure 2. Construction of the gene fusion encoding adhesion peptide and the Linker-CBM. (A) Construction containing one copy of the adhesion peptide at the N-terminal of the CBM; (B) Construction containing two copies of the adhesion peptide. 
$\begin{array}{lllllllll}1 & 2 & 3 & 4 & 5 & 6 & 7 & 8 & 9\end{array}$

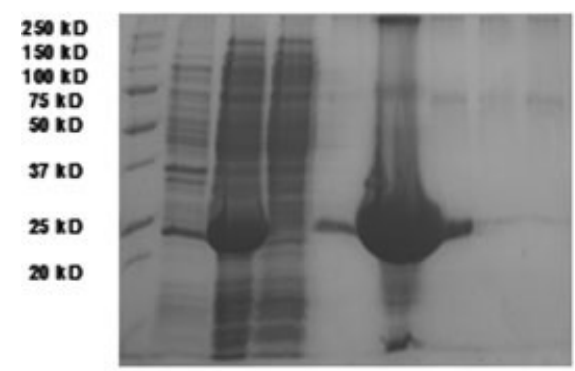

(A)

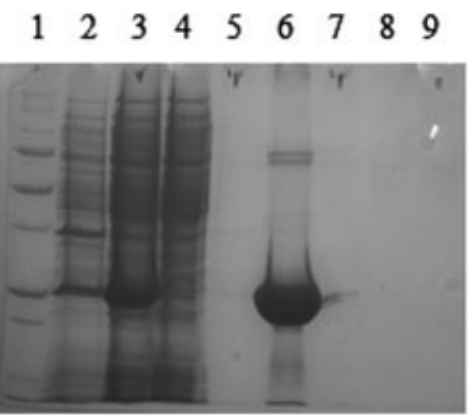

(D) $\begin{array}{lllllllll}1 & 2 & 3 & 4 & 5 & 6 & 7 & 8 & 9\end{array}$

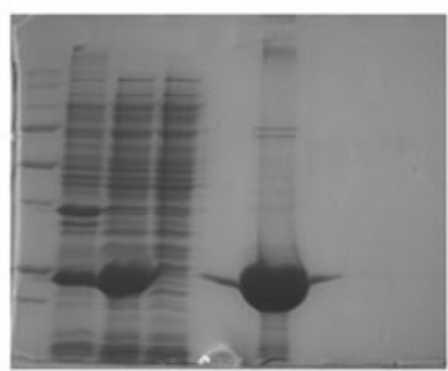

(B)
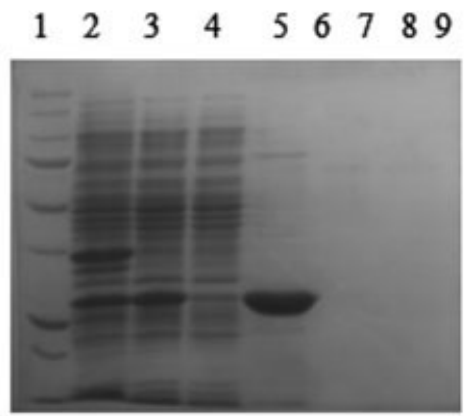

(E) $\begin{array}{lllllllll}1 & 2 & 3 & 4 & 5 & 6 & 7 & 8 & 9\end{array}$

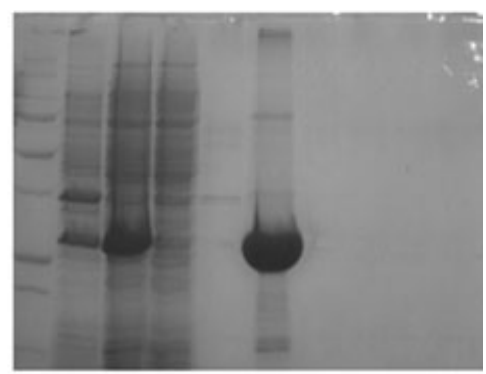

(C)

Figure 3. Analysis by SDS-PAGE of recombinant protein expression and nickel column protein purification. 1-Molecular weight marker (250 kD, $150 \mathrm{kD}, 100 \mathrm{kD}, 75 \mathrm{kD}, 50 \mathrm{kD}, 37 \mathrm{kD}, 25 \mathrm{kD}, 20 \mathrm{kD})$; 2-Insoluble fraction; 3-Soluble fraction; 4Column filtrate; 5 to 9-Eluted fraction with $300 \mathrm{mM}$ of Imidazole. (A) CBM; (B) RGD/CBM; (C) RGD/CBM/RGD; (D) GRGDY/CBM; (E) GRGDY/CBM/GRGDY.

ing to the plate at $4^{\circ} \mathrm{C}$, overnight. Unbound protein was washed out with PBS. In a second test, the adhesion proteins were added to the wells of 24-well polystyrene plates ( $0.25 \mathrm{mg}$ of protein/per well), coated with bacterial cellulose sheets. As referred earlier, the $\mathrm{BC}$ sheets were produced in similar 24-well polystyrene plates, such that they tightly fit in the wells, completely covering the bottom surface. The plates were incubated overnight at $4^{\circ} \mathrm{C}$. Unbound protein was removed and analyzed by SDS-PAGE, and then the bacterial cellulose sheets were washed with PBS. The fibroblasts 3T3 were seeded on the polystyrene plate at a density of $4 \times 10^{4}$ cells/per well, in DMEM medium without serum. One hour after the addition of the cells, the wells were washed with PBS and DMEM with serum $(10 \%)$ was added. The MTS assay and microscope observations of the attached and spreading 3T3 fibroblasts was carried out at $1,5,24$, and $48 \mathrm{~h}$ after the addition of the cells. In the assay with BC, the fibroblasts 3T3 were seeded at a density of $12 \times 10^{4}$ cells/per well, in DMEM medium without serum. The plates were incubated at $37^{\circ} \mathrm{C}$, in atmosphere of $5 \% \mathrm{CO}_{2}$ and $95 \%$ humidified air. Two hours after the addition of the cells, the wells were washed with PBS and DMEM with serum (10\%) was added. The MTS assay and microscope observations of the attachment and spreading of 3T3 fibroblasts were carried out at 2, 24, and $48 \mathrm{~h}$ after the addition of the cells. The results were obtained from at least three different assays, each one with samples in triplicates.

\section{RESULTS}

\section{Production and purification of recombinant proteins}

The gene fragment encoding RGD/CBM was subcloned into the high-expression vector pET 21a, a plasmid vector containing the T7 promoter and a histidine tag sequence. Four different constructs were made, through the fusion of different peptides with a family 3 CBM from the Clostridium thermocellum cellulosome. It has been found, by other authors, that the aminoacids flanking the RGD sequence may affect the affinity of the integrin binding. This interaction may also depend on the peptide exposure, and therefore different constructs were analyzed in this work. Two constructs were made by fusing the RGD or GRGDY sequences at the N-terminal of the linkerCBM. In the other two recombinant constructs a Cterminal linker was designed and an additional RGD or GRGDY sequence was inserted (Fig. 2). As shown in Figure 3, all the recombinant proteins (CBM, RGD/CBM, RGD/CBM/RGD, GRGDY/CBM and GRGDY/CBM/GRGDY) were expressed by E. coli in the soluble fraction. It can also be observed from Fig- 


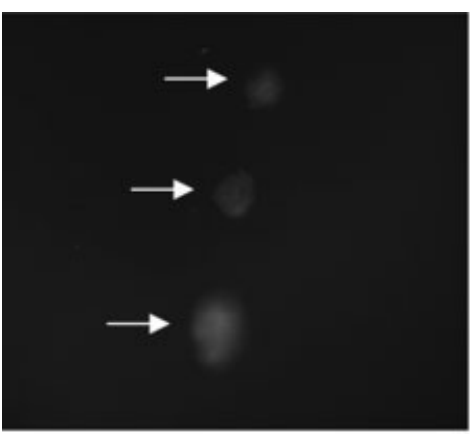

(A)

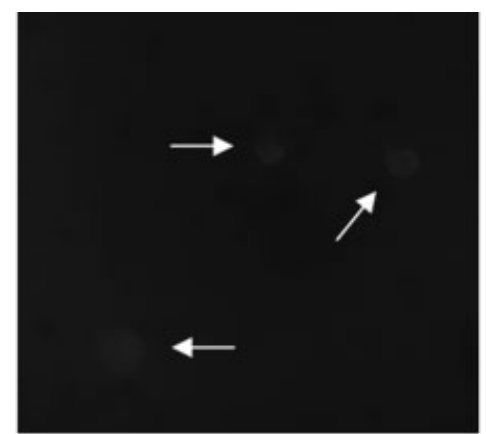

(B)

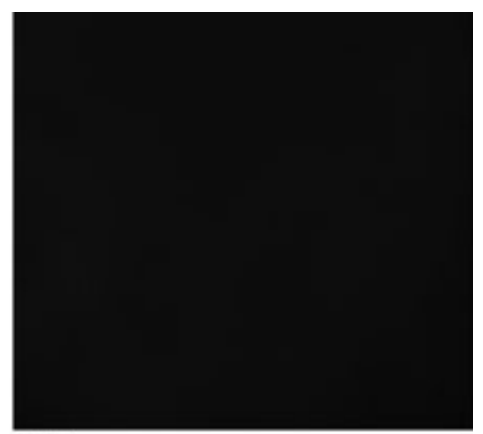

(C)

Figure 4. Fluorescent microscopy showing the binding of recombinant proteins to cell membranes. The arrows point with respect to some of the fluorescent cells. (A) RGD/CBM; (B) GRGDY/CBM; (C) CBM.

ure 3 that the recombinant proteins were isolated to high purity and concentration.

The interaction of RGD and GRGDY with the cells, in medium with or without serum

The fibroblasts, incubated with the recombinant proteins conjugated with FITC, were observed by fluorescence microscopy. This study was performed to evaluate whether the recombinant proteins are able to interact with the cells through the RGD/ GRGDY peptides. When the assays were performed with serum-free medium, only the cells treated with RGD/CBM and GRGDY/CBM became fluorescent, as displayed in Figure 4. When medium containing serum was used, no fluorescent cells were observed, due to competition of the serum proteins with RGD$\mathrm{CBM}$ for the integrins on the cell membranes. These results demonstrate the interaction of the RGD with the cells, and furthermore that the CBM does not interact with the cells.

\section{Attachment of fibroblasts to the polystyrene plate coated with recombinant proteins}

To test the effect of the recombinant proteins in improving the adhesion and proliferation of fibroblasts, the cell culture polystyrene plates, with nonspecifically bound proteins, were used. The polystyrene plates allow the observation of the cells morphology, unlike the bacterial cellulose. In these assays we aimed at observing whether the RGD containing proteins improve the adhesion of fibroblasts and also their effect on the cell differentiation.

The MTS results and microscopic observations showed that the proteins containing the RGD sequence (RGD/CBM and RGD/CBM/RGD) were able to improve the adhesion of fibroblast on polystyrene plates, when compared to the controls (CBM or buffer). Furthermore, the RGD sequence was more effective than GRGDY. The results also showed that, while the protein containing one copy of the GRGDY sequence was able to improve the adhesion of fibroblasts to the polystyrene surface, the protein containing two copies of the GRGDY showed no effect. The fibroblast cells adhered to the wells treated with RGD/CBM and RGD/CBM/RGD showed an elongated morphology one hour after seeding, while the cells on the wells treated with the GRGDY/CBM and GRGDY/CBM/GRGDY and also on the control assays exhibit more spherical morphology (Figs. 5 and 6).

It is quite clear from the results that the presence of the RGD/CBM, RGD/CBM/RGD and, to lower extent, GRGDY/CBM proteins, adsorbed on the polystyrene cell culture wells, improves the adhesion of fibroblasts.

\section{Attachment of fibroblasts on BC surfaces}

The recombinant proteins were added to the bacterial cellulose sheets and left adsorbing overnight. Then, the nonadsorbed protein was removed and analyzed by SDS-PAGE electrophoresis. As may be seen in Figure 7, most of the protein adsorbed on the BC fibers. Thus, not only a functional RGD is present in the recombinant proteins, but also the CBM module fully conserves its functionality and ability to bind cellulose.

The adhesion of fibroblasts to BC was improved by the presence of the adsorbed recombinant proteins. Indeed, the results of the MTS test demonstrated that the proteins containing the RGD sequence were able to significantly increase the adhesion of fibroblast to BC surfaces, while the proteins containing the GRGDY sequence had no effect, taking as reference the cellulose treated with the CBM or with buffer. The results also demonstrated that the protein containing one RGD sequence have a stronger effect than the protein containing two RGD sequences (Fig. 8). 


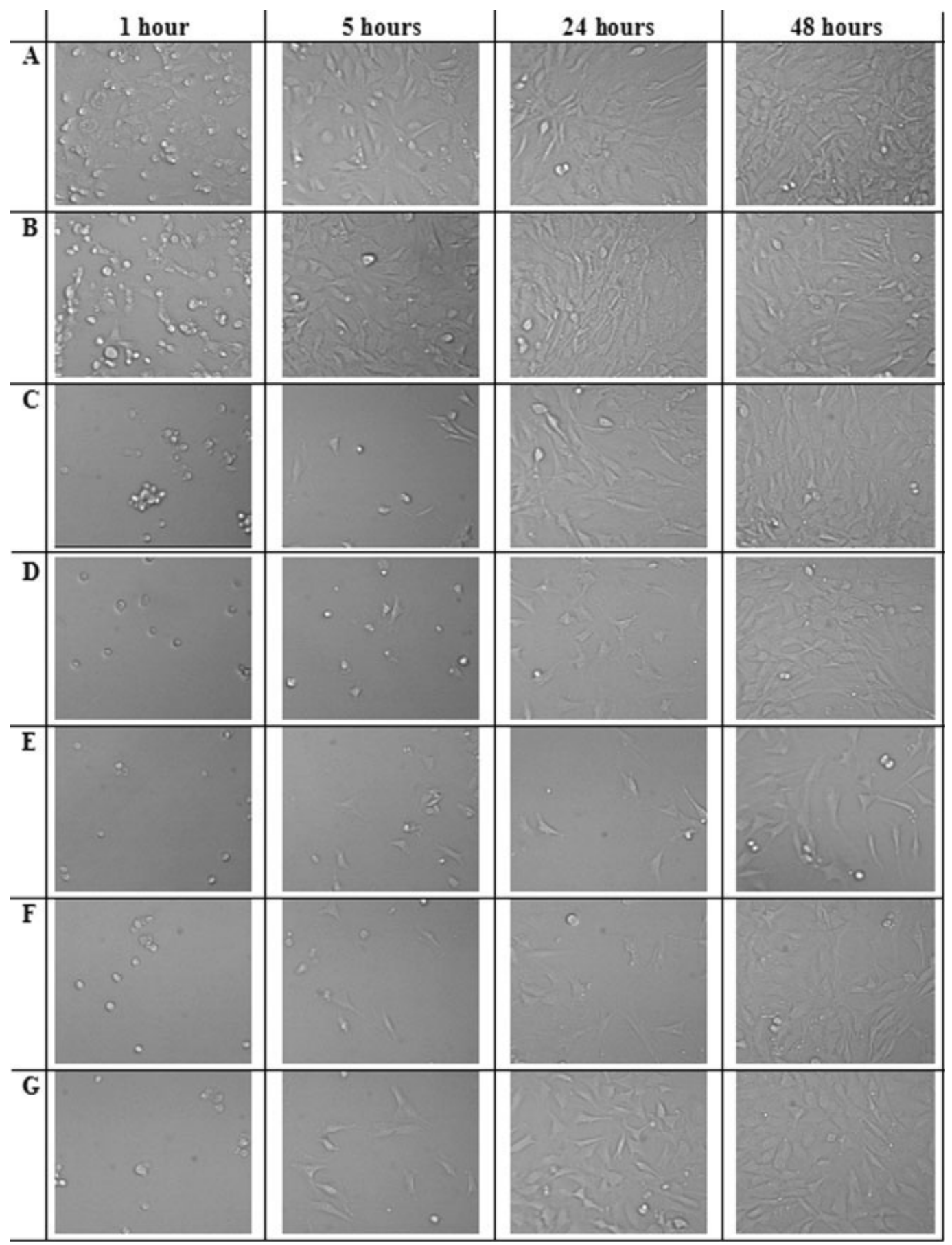

Figure 5. Photographs showing the effect of the recombinant proteins on cell (fibroblasts 3T3) attachment to polystyrene plate. (A) RGD/CBM, (B) RGD/CBM/RGD, (C) GRGDY/CBM, (D) GRGDY/CBM/GRGDY, (E) CBM, (F) Buffer, and (G) Control. The photographs were taken at 1, 5, 24, and $48 \mathrm{~h}$ after addition of cells.

\section{DISCUSSION}

It is known that the amino acids flanking the RGD sequence can influence (positive or negatively) the interaction of the RGD with the integrins on the cell membrane. Many sequences exhibiting different activities have been described: RGDS, GRGDG, GRGDS, GRGDF, YRGDS, CGRGDSPK, and so forth. ${ }^{6}$ Different cell lines recognize the RGD sequences in a distinct way. In this work, the sequences RGD and GRGDY were fused to a CBM, and the interaction of the recombinant proteins with fibroblasts was analyzed.

The observation of fibroblasts, incubated with the soluble recombinant proteins conjugated with FITC, by fluorescence microscopy, revealed that the integ- 


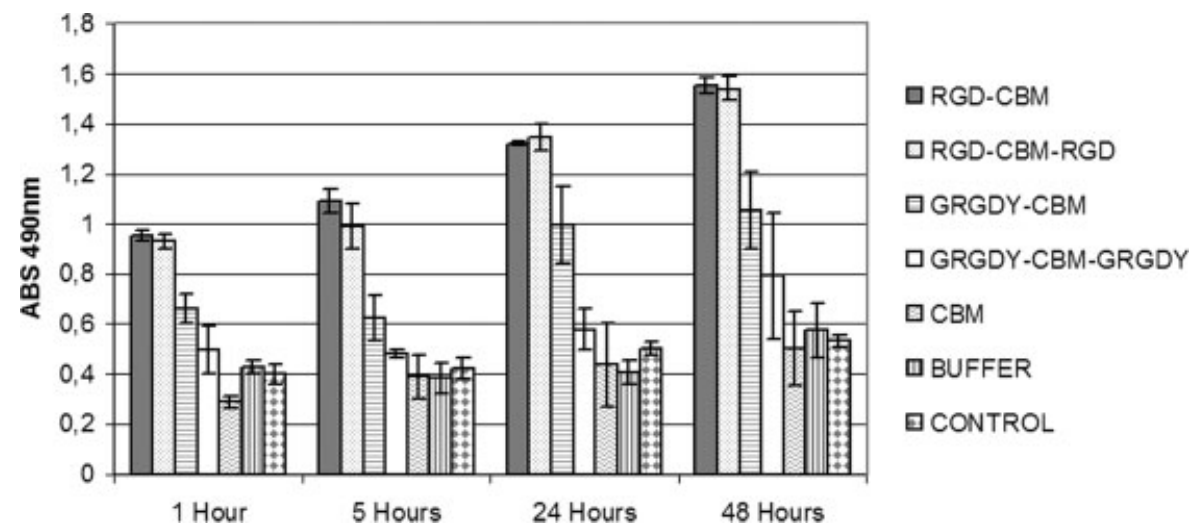

Figure 6. MTS assays of fibroblast culture on polystyrene plates treated with the recombinant proteins (CBM, RGD/ CBM, RGD/CBM/RGD, GRGDY/CBM, and GRGDY/CBM/GRGDY). The MTS test was developed at 1, 5, 24, and 48 $\mathrm{h}$ after addition of cells.

rins interact with the RGD and GRGDY sequences. Different effects are observed in serum and serumfree medium. These differences are probably due to competition of fibronectin and other serum proteins with the RGD-CBM for the integrins on the cell membrane.

The recombinant proteins containing the RGD or GRGDY sequences were able to improve the adhesion of fibroblasts to the polystyrene plate, the CBM alone having no effect. Early cell spreading was also triggered by RGD-CBM and RGD-CBM-RGD proteins, as revealed by the more elongated morphology of the cells one hour after seeding on the polystyrene plates. Similar results have been previously obtained by other authors. Wierzba et al. ${ }^{11}$ produced a recombinant protein containing a RGD sequence at the C-terminus of a CBD from the Cellulomonas fimi endoglucanase A (CenA). This CBD/RGD promoted the attachment of green monkey Vero cell to polystyrene and cellulose acetate. Wang et al. ${ }^{12}$ studied the effect of a protein containing two GRGDS sequences at the N- and C-terminal of a CBD from Trichoderma koningii; this recombinant protein improved the adhesion of keratinocytes and dermal fibroblasts when grafted on the petri dish.

The proteins containing the RGD sequence significantly increased the adhesion of the fibroblast on the cellulose sheet, as compared to the cellulose treated only with CBM or buffer, while the GRGDY had no effect. The protein containing a single RGD had, surprisingly, a stronger effect on the adhesion of fibroblasts than the protein containing two RGDs. Thus, the effects of RGD versus GRGDY and $1 \times$ RGD versus $2 \times$ RGD are distinct, depending on whether the proteins are adsorbed on polystyrene or in bacterial cellulose. The affinity of the cells is expected to

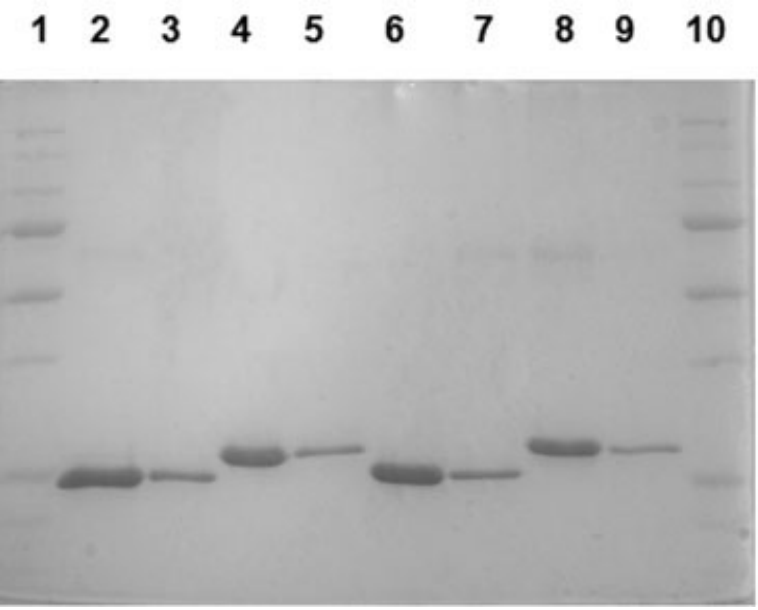

Figure 7. Analysis by SDS-PAGE of the interaction between the recombinant proteins with the cellulose sheets. Line 1, 10, and 11 - Molecular weight marker (250 kD, 150 kD, 100 kD, 75 kD, 50 kD, 37 kD, 25 kD, 20 kD); line 2, 3 - RGD/CBM; line 4, 5 - RGD/CBM/RGD; line 6, 7 - GRGDY/CBM; line 8, 9 - GRGDY/CBM/GRGDY; line 12, 13 - CBM. Lines 3, 5, 7, 9 , and 13 represent the proteins after the interaction with $\mathrm{BC}$ sheets. 


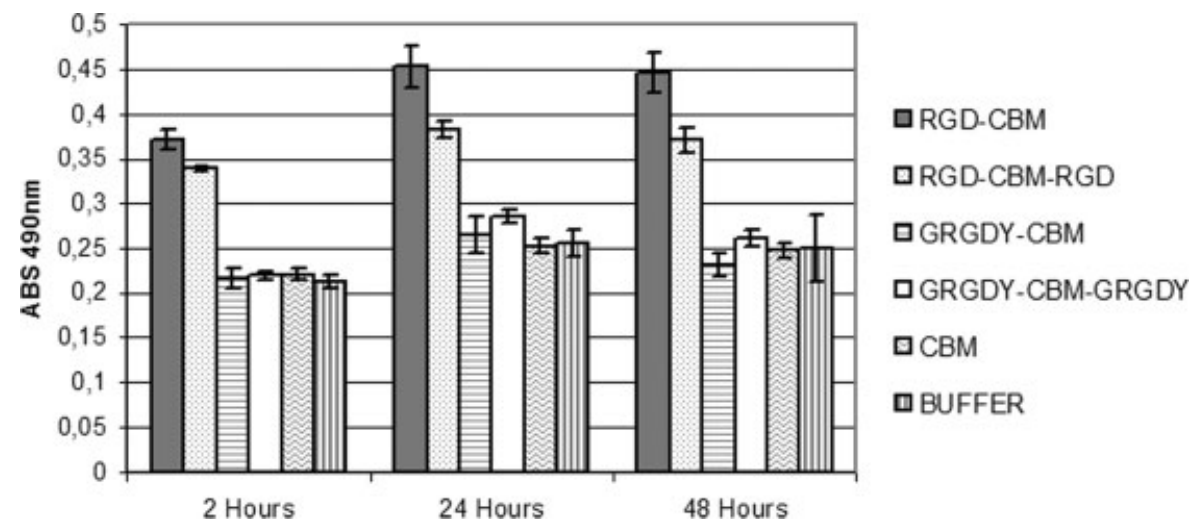

Figure 8. MTS assays of fibroblast culture treated with the recombinant proteins (CBM, RGD/CBM, RGD/CBM/RGD, GRGDY/CBM, and GRGDY/CBM/GRGDY) at the bacterial cellulose pellicles. The MTS test was developed at 2, 24, and $48 \mathrm{~h}$ after addition of cells.

increase with the concentration of RGD copies. This was the motivation for the production of fusion proteins with two RGD copies. However, the results demonstrate that there is not a simple and straight forward relation between the RGD surface concentration and the cells adhesiveness and proliferation. Besides concentration, also the exposure, conformation, and steric effects may play a role to the RGDintegrin interaction. In fact, it seems that the second RGD or GRGDY brings no further functionality to the proteins. It must be remarked that the presence of a RGD sequence, in a protein, does not guarantee cell attachment activity. Indeed, several hundred proteins contain the RGD tripeptide, but most of these do not have cell attachment activity. To be functional, the RGD sequence must be appropriately exposed, in a conformation that can be recognized by a cell surface receptor. ${ }^{11}$ The results obtained in this work consistently showed that, in the assays with $\mathrm{BC}$, the protein containing two RGD sequences had lower activity. The CBM used in this work, from the bacteria Clostridium thermocellum, has N- and C-terminals close to each other. ${ }^{13}$ Probably, the second linker-containing twelve amino acids, followed by the second adhesion peptide and the hexahistidine tag-at the protein Cterminal interferes with the interaction between the N-terminal RGD or GRGDY with the integrins on the cell membrane. Also, it may be that the presence of a hexahistidine tag, following the RGD, interferes with the exposure of this sequence and its interaction with the integrins. This effect is more relevant with cellulose, in which the CBM adsorbs with a specific conformation and orientation, than with the polystyrene plate, in which the nonspecific adsorption probably leads to random orientation of the molecules. Wierzba et al. ${ }^{11}$ observed that different cell lines exhibited different patterns of attachment to CBD/ RGD, depending on whether it was immobilized on polystyrene plate or cellulose acetate. Vero, COS, HFF, 3T3, 293, and U373 cells attached well to CBD/
RGD immobilized on polystyrene or cellulose acetate. CHO, MRC-5, and HEp-2 cells attached to CBD/RGD immobilized on polystyrene, but not to CBD/RGD immobilized on cellulose acetate. BHK and L cells failed to attach to CBD/RGD immobilized on both surfaces. The authors conclude that the adsorption of $\mathrm{CBD} / \mathrm{RGD}$ to polystyrene plate is nonspecific and presents the RGD in a different configuration or environment, and this may account for differences in the observed cell-binding properties, as observed in this work. ${ }^{14}$

\section{CONCLUSION}

Bacterial cellulose is a material with excellent biocompatibility and mechanical properties, thus holding great potential for biomedical applications. In this work, a new approach was developed to functionalize the bacterial cellulose, through recombinant proteins containing adhesion peptides conjugated with a cellulose binding-module. The use of recombinant proteins containing a CBM domain, exhibiting high affinity and specificity for cellulose surfaces, allows the control on the interaction of this material with cells. The CBM may virtually be combined to any biologically active protein for the modification of cellulose-based materials, for in vitro or in vivo applications. The recombinant proteins containing the RGD or GRGDY sequences were cloned and successfully expressed in fusion with a family 3 CBM of Clostridium thermocellum in E. coli expression system. The recombinant proteins containing the adhesion peptide were able to promote adhesion and spreading of the cells. Furthermore, the proteins containing the sequence RGD showed a stronger effect than GRGDY on fibroblast cells. The effect of different adhesive sequences seems to depend on the material where they are adsorbed, 
probably due to conformation effects. Protein models-not in the scope of this work-would probably be useful on helping the design of more effective recombinant proteins.

The author F. K. Andrade is the recipient of a fellowship from Coordenação de Aperfeiçoamento de Pessoal de Nível Superior (CAPES, Brazil) and the author S.M.G. Moreira is the recipient of a fellowship from Fundação para a Ciência e a Tecnologia (FCT, Portugal). The authors wish to acknowledge Prof. Carlos Fontes from Faculdade de Medicina Veterinária da Universidade de Lisboa, Portugal, for providing the pET21 vector with the CBM insert.

\section{References}

1. Goessler UR, Hormann K, Riedel F. Tissue engineering with chondrocytes and function of the extracellular matrix (Review). Int J Mol Med 2004;13:505-513.

2. Plow EF, Haas TA, Zhang L, Loftus J, Smith JW. Ligand binding to integrins. J Biol Chem 2000;275:21785-21788.

3. Van der Flier A, Sonnenberg A. Function and interactions of integrins. Cell Tissue Res 2001;305:285-298.

4. Pierschbacher MD, Ruoslahti E. Cell attachment activity of fibronectin can be duplicated by small synthetic fragments of the molecule. Nature 1984;309:30-33.

5. Pfaff E. Recognition sites of RGD-dependent integrins. In: Eble JA, Kühn K, editors. Integrin Ligand Interactions. R.G. Landes Co.: Austin, Texas; 1997. p 101-121.
6. Hersel U, Dahmen C, Kessler H. RGD modified polymers: Biomaterials for stimulated cell adhesion and beyond. Biomaterials 2003;24:4385-4415.

7. Sakiyama-Elbert SE, Hubbell JA. Functional Biomaterials: Design of novel Biomaterials. Annu Rev Mater Res 2001;31: 183-201.

8. Klemm D, Schumann D, Udhardt U, Marsch S. Bacterial synthesized cellulose-Artificial blood vessels for microsurgery. Prog Polym Sci 2001;26:1561-1603.

9. Czaja WK, Yong DJ, Kawecki M, Brown RM Jr. The future prospects of microbial cellulose in biomedical applications. Biomacromolecules 2007;8:1-12.

10. Tomme P, Boraston A, McLean B, Kormos J, Creagh AL, Sturch K, Gilkes NR, Haynes CA, Warren RA, Kilburn DG. Characterization and affinity applications of cellulose-binding domains. J Chromatogr B 1998;715:283-296.

11. Wierzba A, Reichl U, Turner RFB, Warren RAJ, Kilburn DG. Production and properties of a bifunctional fusion protein that mediates attachment of vero cells to cellulosic matrices. Biotechnol Bioeng 1995;47:147-154.

12. Wang TW, Wu HC, Huang YC, Sun JS, Lin FH. The effect of self-designed bifunctional RGD-containing fusion protein on the behavior of human keratinocytes and dermal fibroblasts. J Biomed Mater Res B Appl Biomater 2006;79:379-387.

13. Tormo J, Lamed R, Chirino AJ, Morag E, Bayer EA, Shoham Y, Steitz TA. Crystal structure of a bacterial family-III cellulose-binding domain: A general mechanism for attachment to cellulose. Embo J 1996;15:5739-5751.

14. Wierzba A, Reichl U, Turner RFB, Warren RAJ, Kilburn DG. Adhesion of mammalian cells to a recombinant attachment factor, CBM/RGD, analyzed by image analysis. Biotechnol Bioeng 1995;46:185-193. 